\title{
IceCube: physics, status, and future
}

\author{
Klas Hultqvist, for the IceCube collaboration \\ Oskar Klein Centre, Dept. of Physics, Stockholm University, 10691 Stockholm, Sweden
}

\begin{abstract}
The IceCube observatory is the first cubic kilometre scale instrument in the field of high-energy neutrino astronomy and cosmic rays. In 2009, following five successful deployment seasons, IceCube consisted of 59 strings of optical modules in the South Pole ice, together with 118 air shower detectors in the IceTop surface array. The range of physics topics includes neutrino signals from astrophysical sources, dark matter, exotic particle physics, cosmic rays, and atmospheric neutrinos. The current IceCube status and selected results are described. Anticipated future developments are also discussed, in particular the Deep Core low energy subarray which was recently deployed.
\end{abstract}

Keywords: IceCube, neutrino astronomy, cosmic rays, south pole, dark matter

\section{Introduction}

At the IceCube observatory, the clear South Pole glacial ice is exploited to detect Cherenkov light emitted by charged particles created in neutrino interactions. An array of digital optical modules (DOMs) equipped with photomultipliers extends throughout a volume of approximately $1 \mathrm{~km}^{3}$, with the central region more densely intrumented. Scheduled for completion in 2011, IceCube allows the detection of neutrino interactions above a few tens of $\mathrm{GeV}$, with a maximum sensitivity in the $\mathrm{TeV}-\mathrm{PeV}$ range.

Extraterrestrial neutrinos are expected from a variety of sources. Generically, high energy cosmic ray particles will give rise to neutrinos in their interactions with matter or radiation, either in the immediate vicinity of the acceleration sites or while traveling through space. It is thus possible to estimate the neutrino flux that corresponds to the observed flux of cosmic rays [1]. Such considerations have dictated sensitivity requirements which translate into a cubic kilometre detector.

Owing to their low interaction cross-section and lack of electric charge, neutrinos propagate over vast distances, quite undisturbed by matter, radiation, and magnetic fields. This is in contrast to photons, which can be absorbed by matter, and which cannot travel cosmological distances if their energy is above the $\mathrm{TeV}$ range, because of absorption by background radiation. Similarly, protons of the highest energies can be absorbed through the GZK mechanism [2], while at lower energies they are deflected by magnetic fields and do not point back to the source. It is therefore possible that there are "hidden" sources which can only be detected via their neutrino emission. In cases where emission of gamma rays or high energy cosmic rays is seen, a detection of neutrinos could elucidate the nature and location of cosmic ray accelerators. (Gamma rays from $\pi^{0}$ decay would be accompanied by neutrinos from $\pi^{+} / \pi^{-}$ decay.) Promising candidate acceleration sites are relativistic ejecta from active galactic nuclei (AGNs) or gamma-ray bursts
(GRBs). There are also objects within our galaxy, such as supernova remnants, pulsar winds, or microquasars, in which acceleration processes could yield neutrino emission.

Even if individual neutrino sources are not seen, their combined diffuse flux may stand out above the atmospheric neutrino flux. The study of the atmospheric neutrino flux is therefore an important topic. It provides a useful calibration point and may in addition yield results relevant to the intrinsic properties of neutrinos.

There are abundant indications of dark matter in the form of non-relativistic weakly interacting massive particles (WIMPs) [3]. These could constitute a class of "hidden" sources, detectable via neutrinos, as they annihilate in the centre of the Sun or other dense regions [4, 5]. Promising candidate WIMPs are the neutralinos of supersymmetric models [5] or Kaluza-Klein excitations in models of extra dimensions [6].

Section 2 describes the IceCube detector and its performance, and selected analysis results are discussed in section 3. Future enhancements are discussed in section 4

\section{The IceCube detector}

Figure 1 illustrates the layout of the IceCube observatory in the polar icesheet. Also shown is the AMANDA array [7], which continued to operate as a part of IceCube until it was finally closed down in May 2009. In addition to the deep ice detector, there is a surface array, IceTop, for detecting cosmic ray air showers. Each IceTop station is located above a string, and consists of two tanks. Each tank is filled with clear ice and monitored by two DOMs running at different gains to achieve the required dynamic range.

IceCube construction takes place during the austral summer. A $5 \mathrm{MW}$ hot-water drilling system is used to melt holes in the ice down to a depth of $2,45 \mathrm{~km}$. In each hole a string consisting of a cable carrying 60 optical modules is deployed, and the water in the hole then re-freezes. During the recent deployment 


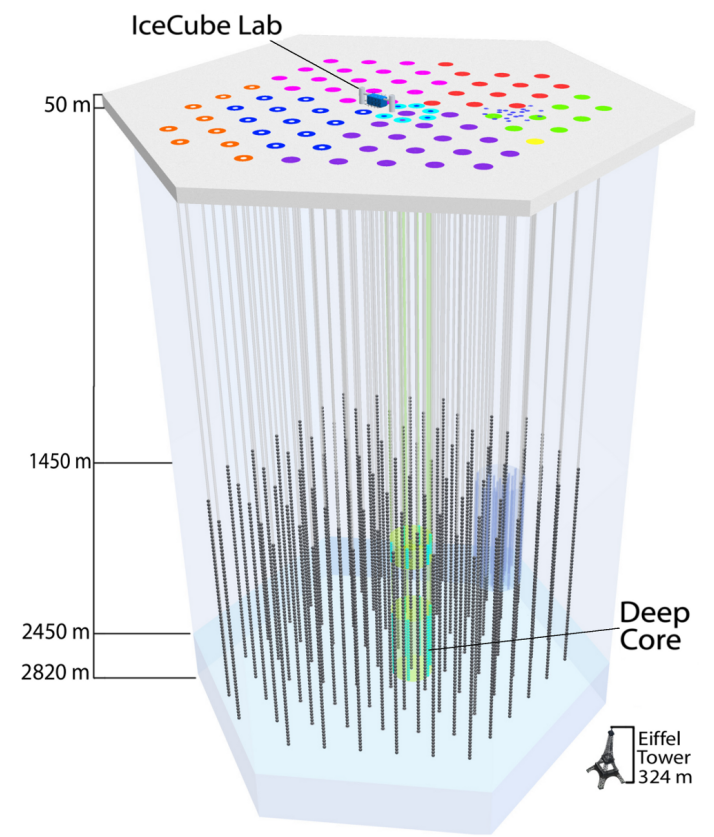

Figure 1: The IceCube detector. Filled circles indicate the 59 strings completed for the 2009 data taking season. The shaded region near the centre is the Deep Core subarray, and the one to the right shows the position of AMANDA.

season, the time between completed deployments was about two days. When completed in 2011, IceCube will consist of at least 80 strings and 80 IceTop stations. Fifty-nine strings were in operation during 2009, and an additional 20 were deployed in the summer season of 2009/2010.

Figure 2 shows an IceCube optical module [8]. The lightsensitive element is a $25 \mathrm{~cm}$ photomultiplier tube (PMT), looking down. This is optically coupled to a surrounding pressure sphere via transparent gel. The sphere also contains a highvoltage generator, 12 LED flashers for calibration purposes, communication and time-calibration electronics, and two different types of digitization hardware. Three Analogue Transient Waveform Digitizer (ATWD) channels with different gains are used to digitize the PMT output with nanosecond precision during $400 \mathrm{~ns}$. There is also a fast ADC running at $40 \mathrm{MHz}$ during $6,4 \mu \mathrm{s}$. The DOM is triggered by a discriminator coupled directly to the PMT output, and the input to the digitizers is delayed by $75 \mathrm{~ns}$ to allow the capture of the complete waveform.

An important component in the IceCube detector is the ice itself. Its optical properties have been measured in situ using light emitting devices [9], and the ice model and its implementation in light propagation and reconstruction software are being gradually refined. Scattering dominates over absorption, with an overall attenuation length in excess of $20 \mathrm{~m}$ in the upper regions of the detector, and about twice that value below a depth of $2100 \mathrm{~m}$. In between these two regions there is a dusty layer with strong scattering and absorption.

IceCube is designed to detect neutrinos of all flavours and distinguish extraterrestrial neutrino signals from the background. The remainder of this section contains a discussion



Figure 2: A schematic drawing of an IceCube optical module (DOM). Figure from reference 8

of the principles involved, as well as some examples of detector performance. In all cases, detection relies on the emission of Cherenkov light, at an angle of $41^{\circ}$ from the direction of propagation of charged relativistic particles.

When a muon from a charged current (CC) $v_{\mu}$ interaction passes through the instrumented volume it gives rise to a characteristic "track" consisting of large pulses in DOMs close to the muon trajectory, separated in time by the muon flight time. Its direction can therefore be well determined. The point spread function for well reconstructed muon neutrino events is shown in Figure 3. Sub-degree resolution has been achieved already with the 40 -string configuration. Muons which pass close to

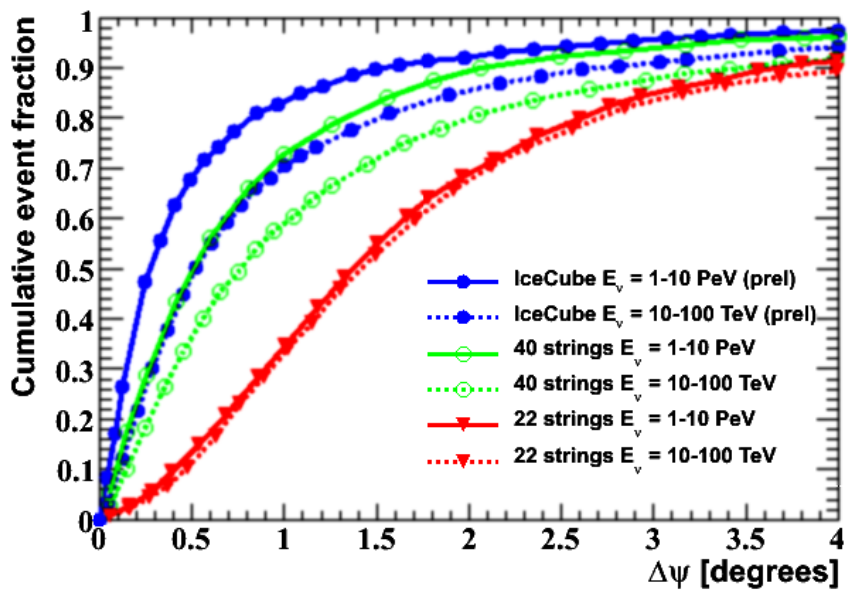

Figure 3: The cumulative point spread function for well reconstructed events, including the angle between the incoming neutrino and the muon. The improvement between the 22- and 40 string case is not only due to the larger detector, but also to an improved reconstruction algorithm. Figure from reference 10

the edge of the instrumented volume, or outside, can also be detected, especially at high energies. In this case, however, the event reconstruction and background rejection is more difficult, 
and relies more heavily on the description of the emission of Cherenkov light and its propagation through the ice.

To achieve sensitivity to extraterrestrial neutrino sources, one must also eliminate the overwhelming background of downgoing muons from cosmic ray air showers. The traditional approach is to use the Earth as a filter and select up-going events. There will still be a large background from downgoing muons which are mis-reconstructed as upgoing. By requiring well reconstructed events, however, one can define a clean sample of up-going neutrinos, as illustrated in Figure 4

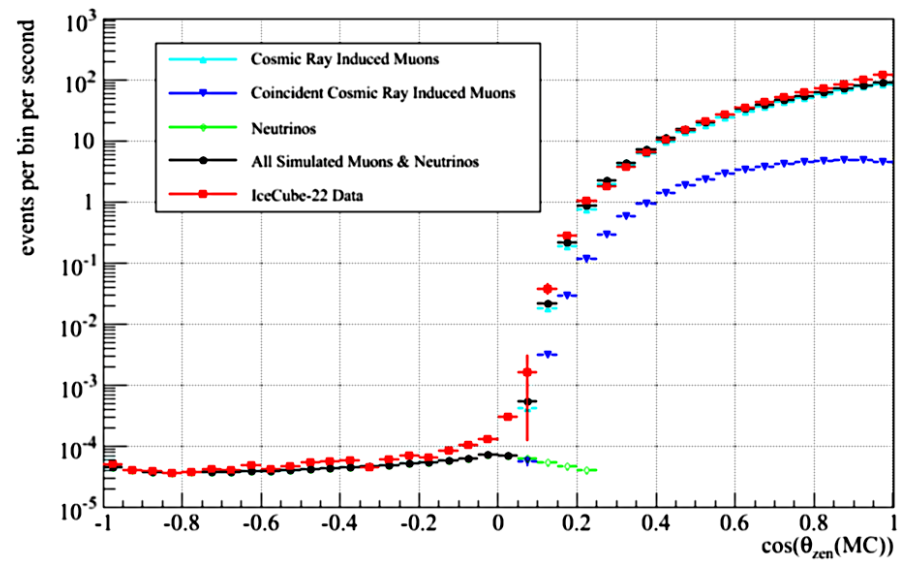

Figure 4: Muon trigger rates in the 22-string IceCube configuration, as a function of the cosine of the zenith angle. The rate in data (red squares) is compared to a simulation including both atmospheric muons and muons from interacting atmospheric neutrinos. Requirements to select well reconstructed events were applied, and the rates are corrected for the efficiency of these. Figure from reference 11

The energy spectrum of atmospheric neutrinos falls rapidly, as $\sim E^{-3,7}$, whereas neutrinos from atrophysical sources are expected to have a much harder spectrum $\left(\sim E^{-2}\right)$. An energy estimator will therefore provide additional discrimination in the search for extraterrestrial point sources. Furthermore, it is essential in the search for a diffuse signal of extraterrestrial muon neutrinos. For muons above a few $\mathrm{TeV}$, the amount of Cherenkov light scales roughly linearly with energy, and the total number of triggered DOMs has been used as an energy proxy. Recently, a more direct method for measuring the energy loss along the muon trajectory has been developed [17, 18], with a resolution in $\log _{10}\left(E_{\mu} / \mathrm{GeV}\right)$ of around $30 \%$ in the range between $1 \mathrm{TeV}$ and $100 \mathrm{PeV}$, see Figure 5 .

Two recent observations of downgoing atmospheric muons illustrate that the reconstruction methods work. One is the detection of the reduction of the cosmic ray flux caused by absorption in the Moon [12], see Figure 6. Systematic biases in the reconstruction could affect the position of the minimum. No such effect is seen in the analysed data. Increased statistics will render the test more stringent. The other result is the observation of a global anisotropy in the cosmic ray arrival directions [13]. This is the first such measurement in the Southern Hemisphere, and it agrees well with measurements done in the North [14, 15].

Neutral current interactions give cascade events in which no particle travels a distance comparable to the spacing between

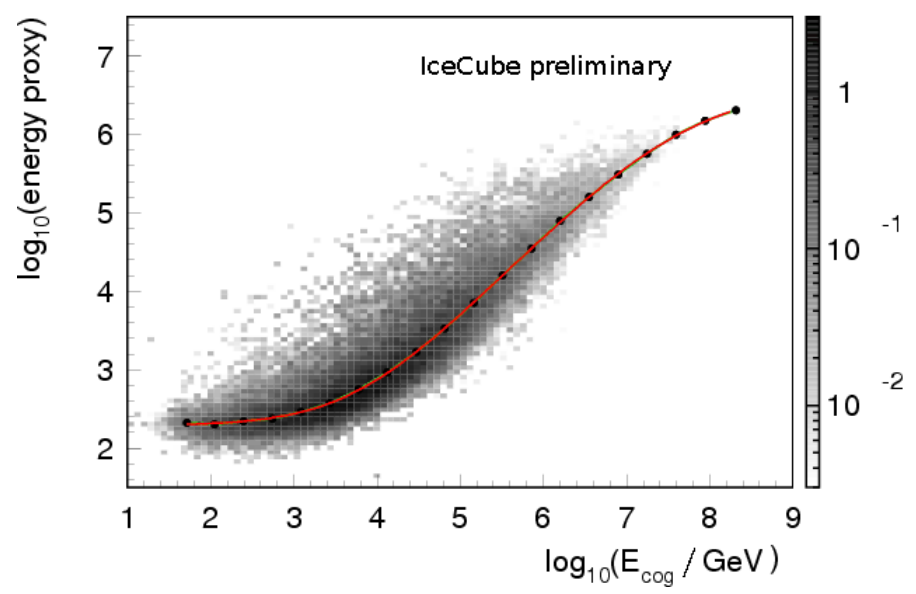

Figure 5: Reconstructed vs true logarithm of the muon energy, at the point of closest approach to the centre-of-gravity of hit modules. Note the logarithmic intensity scale.



Figure 6: Angular distance from the Moon for events in a $1,25^{\circ}$ declination band centered on the Moon. A 5,2 $\sigma$ deficit is seen in the central bin. The figure is based on eight months of data taken with the 40-string configuration in 2008.

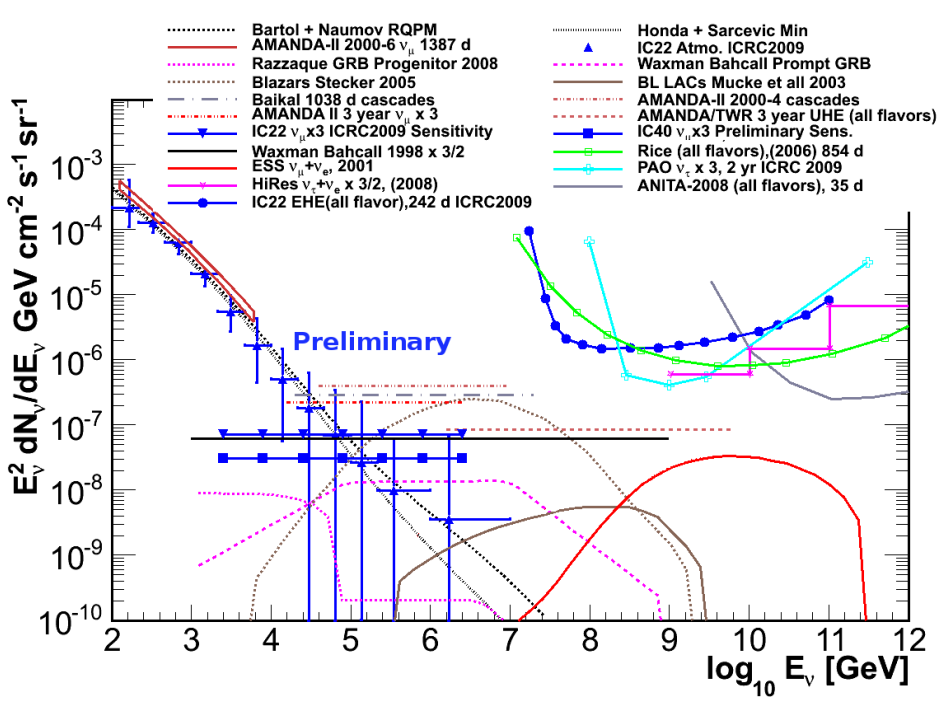

Figure 7: Diffuse neutrino flux predictions, measurements, and limits. All results are scaled to apply to the total neutrino flux, assuming that the fluxes are equal for all three flavours. See text for details. 
detector modules. This is also true for $v_{\mathrm{e}} \mathrm{CC}$ events below the EeV range, and for $v_{\tau} \mathrm{CC}$ events below a few PeV. For such cascades, the light is emitted from a small region, but preferentially at the Cherenkov angle of $41^{\circ}$ with respect to the shower axis. Making use of this information, and detailed knowledge about light propagation in the ice, an angular resolution of 30 to 35 degrees is expected for cascades in the energy range between $10 \mathrm{TeV}$ and $10 \mathrm{PeV}$ [16]. The resolution in $\log _{10}(E / \mathrm{GeV})$ is $\sim 13 \%$ for cascades contained in the instrumented volume [16]. As the array grows, the volume where a cascade can be identified increases faster than the detector volume, and much faster than the area exposed to a throughgoing muon. Therefore the cascade channel, with its better energy resolution, is becoming more important as the detector grows.

At higher energies electromagnetic showers initiated by electrons from $v_{\mathrm{e}}$ interactions become elongated owing to the LPM effect [19, 20], which makes a better angular reconstruction possible [21]. For $v_{\tau}$ energies in the $\mathrm{PeV}$ range and above, the distance travelled by the $\tau$ from a CC interaction leads to several signatures [22]. These are of special interest as there is no terrestrial $v_{\tau}$ background flux, whereas astrophysical neutrino fluxes are expected to contain a mix of all flavours.

\section{Selected results}

Figure 7 shows two preliminary results on the diffuse neutrino flux, obtained from the IceCube data taken in 2007 with 22 strings (IC22). The triangles with error bars show a preliminary energy spectrum, obtained using an unfolding method [17]. Agreement is found with previous AMANDA unfolding results, and with the calculated atmospheric neutrino flux. The connected filled circles show a quasi-differential limit 1 at $90 \%$ confidence level, obtained in a preliminary low background search for Extremely High Energy (EHE) events [23]. No candidates were found.

Also shown are projected sensitivities of the searches with IC22 and IC40 (the 2008 40-string configuration) for extraterrestrial diffuse fluxes falling as $E^{-2}$ [18]. They are drawn as constant lines with a length corresponding to a region which would contain $90 \%$ of the selected signal events. The figure also shows results obtained with AMANDA. These are an unfolded energy spectrum for atmospheric neutrinos, based on seven years of muon data [24], limits on a diffuse $E^{-2}$ flux obtained using muons [25] and cascades [26], and a limit on the flux of Ultra High Energy (UHE) neutrinos based on three years of AMANDA data [27].

Figure 7 also includes some results from other detectors. They are, in order of increasing energy, the Lake Baikal neutrino observatory, the RICE South Pole radio Cherenkov array, the Pierre Auger Observatory (PAO), the HiRes air fluorescence experiment, and the ANITA radio Cherenkov detection antenna. Also shown are the Waxman-Bahcall flux derived

\footnotetext{
${ }^{1}$ This is the limit on a spectrum which falls as $E^{-2}$ over one decade in energy, and is zero outside this range.
}

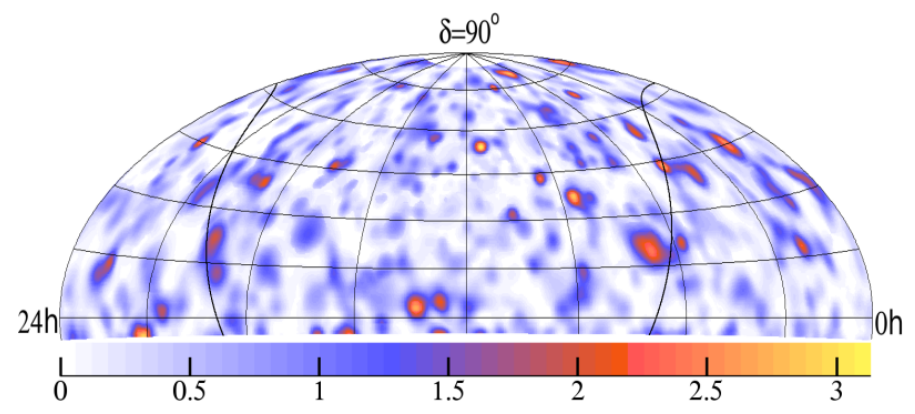

Figure 8: Significance map showing $-\log _{10} p$ for points in the northern sky, based on a search for point sources in seven years of AMANDA data.

from the observed cosmic ray spectrum [1] and calculated contributions to the diffuse neutrino fluxes from atmospheric neutrinos, gamma-ray bursts [28], active galactic nuclei [29], and the GZK process [30].

Some of the neutrino sources contributing to a diffuse flux may be individually seen as point sources. Since the energy spectrum of the signal is expected to be harder than that of the atmospheric neutrino background, a likelihood ratio hypothesis test including energy and direction is the natural approach [31]. Repeating this for a very fine grid of source directions gives a map of the $p$-value (the a priori probability, in the absence of a source, to obtain a result which is at least as signal-like as the one actually obtained).

Figure 8 shows a sky map obtained from seven years of AMANDA observations [32]. The maximum significance is at $\delta=54^{\circ}, \alpha=171^{\circ}$, where the $p$-value is $p=7,4 \cdot 10^{-4}$. The probability of obtaining such a $p$-value or lower somewhere in the sky, determined by randomising the right ascension, is $p=95 \%$. Hence there is no indication of a point source signal.

The point source search in the data taken with the IceCube 22-string configuration [33] was about twice as sensitive as the AMANDA one, and showed a conspicuous deviation from background with $p=7 \cdot 10^{-7}$ at $\delta=11,4^{\circ}, \alpha=153,4^{\circ}$. However, the final $p$-value, accounting for the multiple trials in scanning the sky, is $p=1,3 \%$, which is a very marginal significance.

A point source analysis of six months of data taken with 40 strings (out of 13 months in total) [10] yielded the sky map shown in Figure 9 . Here the most significant point is at $\delta=15,4^{\circ}, \alpha=115,0^{\circ}$ and the $p$-value, accounting for multiple trials, is $61 \%$. The map includes the southern sky, where the background of downgoing muons completely dominates the atmospheric neutrinos. A zenith dependent cut on the muon energy parameter was imposed to ensure a nearly uniform zenith distribution of the background. As a result, the search in the southern hemisphere is sensitive primarily to neutrino energies in the $\mathrm{PeV}$ range, as compared to $\mathrm{TeV}$ energies for the northern hemisphere. The better angular resolution for higher energy can be clearly seen in the figure. A search above the horizon has also been performed using the 22-string data [34].

In each of the point source analyses mentioned above, source candidates on a pre-defined list have been tested, in addition to the very fine grid search. The most significant result is for the Geminga pulsar in the seven-year AMANDA data $(p=0,9 \%)$. 


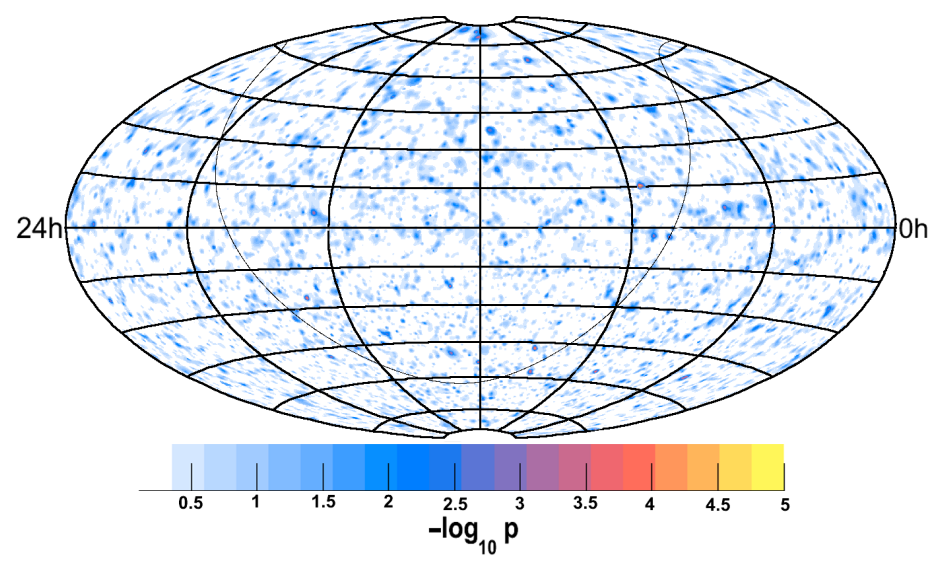

Figure 9: Significance map for a point source search in six months of data taken with the IceCube 40-string configuration. There are 6796 events from the northern celestial hemisphere, and 10981 from the southern one, where the atmospheric muon background dominates.

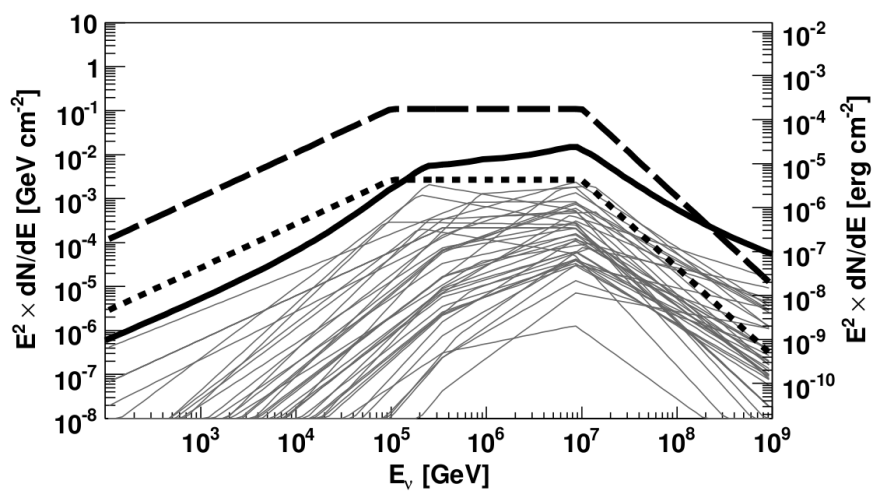

Figure 10: Calculated prompt neutrino fluences for 41 GRBs searched for neutrino emission in the 22-string data. Also shown is the sum over all bursts (thick solid line), the Waxman-Bahcall spectrum [42] for a single burst (thick dotted) and for 41 bursts (thick dashed)

For the IceCube searches, however, Geminga gives downward fluctuations $(p>0,5)$. The point showing the excess in the 22string data was included as a candidate in the 40-string search, where it showed a downward fluctuation.

A search for time clustering of events from candidate sources has also been carried out using the 22-string data [35], and there have been efforts to correlate IceCube observations in time with flares observed in the electromagnetic spectrum [36]. There is also a programme to provide online triggers for air Cherenkov telescopes [37], and for fast optical follow-up observations of transient neutrino emission, as expected from e.g. supernovae or gamma-ray bursts [38].

In addition, the recorded data are searched for neutrino events coincident in time and direction with GRBs detected by satellites [39, 40, 41]. The approach has recently been refined to model the emission of individual bursts based on the observed parameters, as shown in Figure 10. Limits obtained [39] are two orders of magnitude above the fluence sum in Figure 10 . and about a factor five above a predicted lower energy precur-

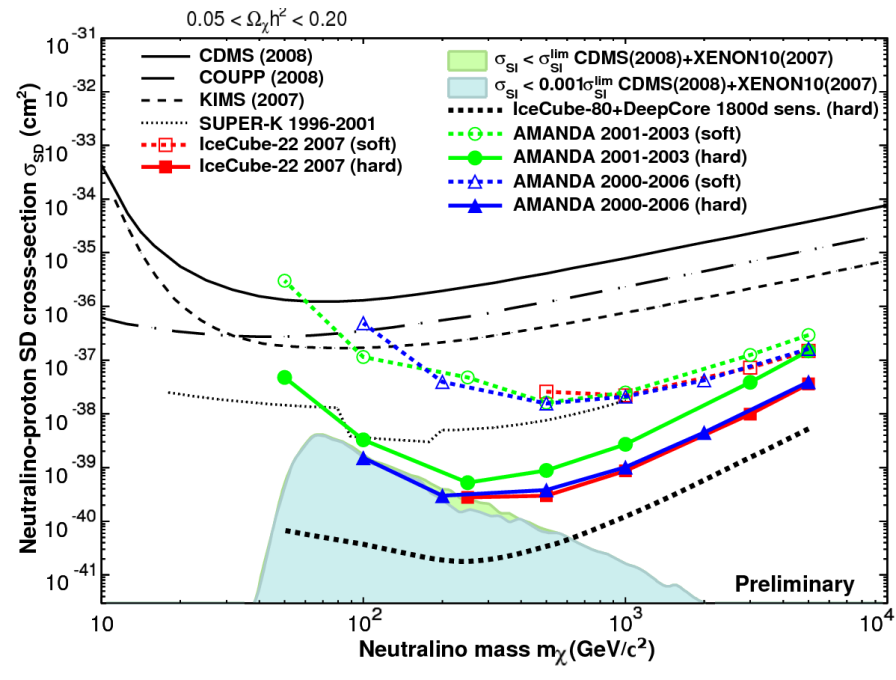

Figure 11: Limits on the spin-dependent neutralino cross-section obtained with IceCube and AMANDA compared to results from Super-Kamiokande and direct detection experiments (cited in reference 43). Also shown is the region corresponding to allowed supersymmetric models found using DarkSusy [45] for current limits on the spin-independent cross-section, and the region which would still be allowed if these limits improved by a factor 1000 .

sor fluence[28]. A study was also made [39] of the expected sensitivity of the full IceCube detector, with future GRB samples from the Fermi and Swift satellites. It indicates that fluences, predicted from the assumption that GRBs are the dominant sources of ultra-high energy cosmic rays, will be detected or ruled out after some years of running IceCube [39].

In conclusion, no evidence for steady or transient neutrino point sources has been found so far.

The indirect detection of dark matter via neutrinos from WIMP annihilations is another important aim of IceCube. The nearest candidate sites for gravitational trapping are the centres of the Earth and of the Sun. Since the light elements dominate the Sun, the spin-dependent scattering cross-section is more relevant for WIMP capture in the Sun than in the Earth. For the same reason, searches for an indirect signal from capture in the Sun complement those in direct detection experiments, which are primarily sensitive to the spin-independent cross-section.

The 22-string IceCube data taken with the Sun below the horizon have been analysed, using a likelihood ratio method involving the angle relative to the Sun, to test hypotheses of neutralino annihilations [43]. Neutralino masses in the range from $250 \mathrm{GeV} / c^{2}$ to $5 \mathrm{TeV} / c^{2}$ were selected, and two extreme annihilation channels were investigated, the "hard" $\left(\mathrm{W}^{+} \mathrm{W}^{-}\right)$and the

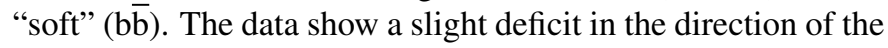
Sun [43]. Following the procedure of reference 44, the resulting limits on the muon flux in the ice were converted into the limits on the spin-dependent cross-section shown in Figure 11. The figure also shows limits obtained using AMANDA data [46]. One set was obtained using the data sample and techniques from the seven-year point source search [32], and another from a dedicated analysis of three years of data [47]. It can also be seen from Figure 11 that the limits on the spin-independent cross-section from direct detection searches do not appreciably 


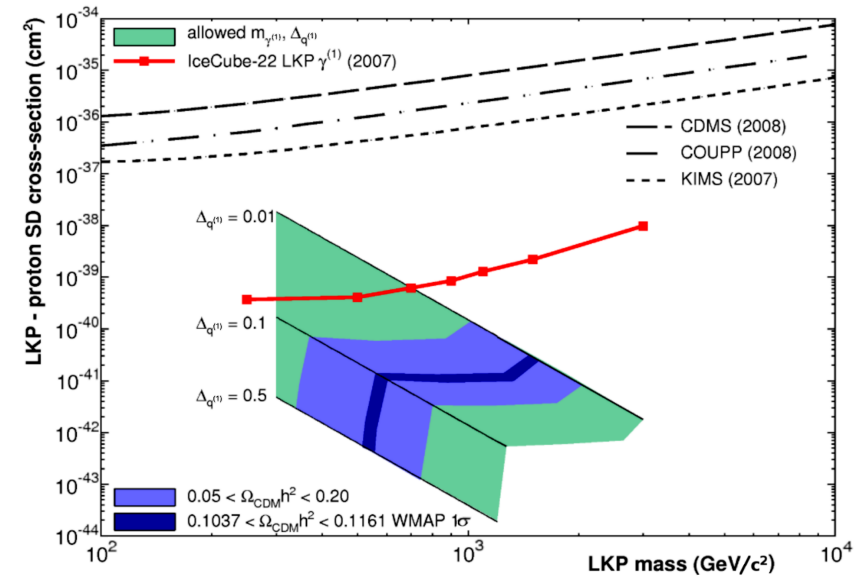

Figure 12: Limits on the spin-dependent cross-section of the lightest KaluzaKlein particle (LKP) and the region allowed in minimal models of extra dimensions. $\Delta_{q^{(1)}}$ is the relative mass splitting between the first quark excitation and the LKP. The dark narrow (blue) region is obtained when consistency with the relic density inferred from WMAP measurements [49] is required within one sigma.

constrain the supersymmetric model space in this projection.

The negative result of the search in the IceCube 22-string data was also interpreted [48] in the framework of Kaluza-Klein theories with universal extra dimensions, with the WIMP annihilating in the Sun being $\mathrm{B}^{(1)}$, the first excitation of the weak hypercharge gauge boson. Figure 12 shows the cross-section limit obtained, and the region allowed in minimial models. The limit from the seven-year AMANDA point source sample is almost identical.

\section{Future}

There are several initiatives to benefit from the presence of the IceCube array and extend the range of physics topics which can be addressed.

The IceCube volume is too small to collect a sizeable sample of neutrino events at EHE energies, because of the low flux. Acoustic and radio emissions from neutrino interactions are attenuated much less than light, and could be used to increase the effective volume at high energies in a hybrid approach [50].

The South Pole Acoustic Test Setup [51] has been used to study sound propagation and the acoustic environment in the ice using pingers and receivers deployed in IceCube holes. The preliminary results indicate an attenuation length of around $300 \mathrm{~m}$, which is more than an order of magnitude below the predicted value [52].

Within IceCube there has also been a successful development programme, AURA, to test methods for radio detection in the ice [53]. A proposal for an $\sim 80 \mathrm{~km}^{2}$ array (Askaryan Radio Array, ARA) has been submitted. In addition, the possibility of a surface radio array to detect air showers was studied [54].

At the other end of the energy scale, WIMP annihilations would probably give neutrinos in the energy range from $\mathrm{GeV}$ to $\mathrm{TeV}$. The original IceCube design is not optimal for these energies. Additional funding was secured, however, for six strings

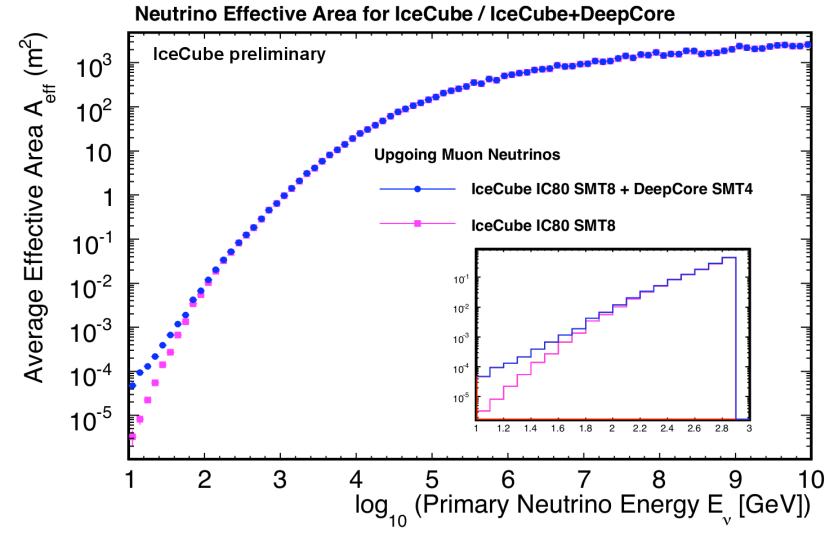

Figure 13: The effective area for muon neutrino detection at trigger level of the originally planned 80 -string array, and with the addition of the Deep Core

added at the centre of IceCube. These are equipped with PMTs with enhanced quantum efficiency, about $30 \%$ higher than that of standard DOMs. The modules are placed closer to each other than on standard strings ( $7 \mathrm{~m}$ as compared to $17 \mathrm{~m}$ ). The instrumented region is between $2100 \mathrm{~m}$ and $2450 \mathrm{~m}$ (below the main dust layer), where the exceptionally clear ice, together with the denser instrumentation, contributes to an overall ten-fold increase in photon collection efficiency. The lower portions of the central thirteen strings, including the aforementioned six, constitute the Deep Core subarray [55], with a fiducial mass of about 13 MT. It is surrounded by at least three layers of instrumentation on all sides. Simulations indicate that an algorithm rejecting events with early hits outside the Deep Core will be able to reduce the atmospheric muon background by a factor of $10^{6}$, bringing it down to the level of atmospheric neutrinos [56].

Figure 13 shows the IceCube effective area with and without the additional Deep Core strings. The improvement is significant at low energies. The thick dotted line in Figure 11 shows the expected sensitivity in the solar WIMP search ten years after the completion of IceCube, using data taken with the Sun below the horizon. The sensitivity below $100 \mathrm{GeV} / c^{2}$ is almost exclusively due to the Deep Core.

The physics potential of the Deep Core goes well beyond the search for Dark Matter [57]. The possibility to reject atmospheric muons entering through the surrounding detector array will make it possible to study neutrino emission from the southern hemisphere without requiring PeV energies. Furthermore, the atmospheric neutrino spectrum in the range between $10 \mathrm{GeV}$ and $1 \mathrm{TeV}$ will be accessible, which will make it possible to conduct high-statistics studies of neutrino oscillations at $\sim 10 \mathrm{GeV}$ over baselines comparable to the size of the Earth. It will be possible to study $v_{\mu}$ disappearance at higher energies than previously done, and possibly $v_{\tau}$ appearance. It has also been suggested that the Deep Core might have some sensitivity to the neutrino mass hierarchy [58].

The additional six Deep Core strings were successfully installed during the 2009/2010 deployment season together with 14 standard IceCube strings, bringing the total to 79 strings. 


\section{Acknowledgements}

We acknowledge the support from the following agencies: U.S. National Science Foundation-Office of Polar Program, U.S. National Science Foundation-Physics Division, University of Wisconsin Alumni Research Foundation, U.S. Department of Energy, and National Energy Research Scientific Computing Center, the Louisiana Optical Network Initiative (LONI) grid computing resources; Swedish Research Council, Swedish Polar Research Secretariat, Swedish National Infrastructure for Computing, and Knut and Alice Wallenberg Foundation, Sweden; German Ministry for Education and Research (BMBF), Deutsche Forschungsgemeinschaft (DFG), Research Department of Plasmas with Complex Interactions (Bochum), Germany; Fund for Scientific Research (FNRS-FWO), FWO Odysseus programme, Flanders Institute to encourage scientific and technological research in industry (IWT), Belgian Federal Science Policy Office (Belspo); Marsden Fund, New Zealand.

[1] E. Waxman and J.N. Bahcall, Phys. Rev. D 59 (1999) 023002, arXiv:hepph/9807282

[2] K. Greisen, Phys. Rev. Lett. 16 (1966) 748;

G. T. Zatsepin and V. A. Kuzmin, JETP Lett. 5 (1966) 78.

[3] G. Bertone, D. Hooper, J. Silk, Phys. Rept. 405 (2005) 279: D. Hooper, arXiv:0901.4090

[4] J. Silk, K. Olive, M. Srednicki, Phys. Rev. Lett. 55 (1985) 257;

[5] G. Jungman, M. Kamionkowsi and K. Griest, Phys. Rept. 267 (1996) 195, and references therein.

[6] D. Hooper and S. Profumo, Phys. Rept. 453 (2007) 29, arXiv:hep$\mathrm{ph} / 0701197$

[7] AMANDA Collaboration: E. Andrés et al., Astropart. Phys. 13 (2000) 1; Nature 410 (2001) 441; W. Wagner et al., AMANDA coll.: New Capabilites of the AMANDA-II High Energy Neutrino Detector, Proc. 28th Int. Cosmic Ray Conf., Tsukuba, Japan, July 2007.

[8] IceCube collaboration: R. Abbasi et al., Nucl. Instrum. Meth. A 601 (2009) 294-316, arXiv:0810.4930

[9] M. Ackermann at al., J. Geophys. Res. 111 (2006) D13203; N. E. Bramall at al., Geophys. Res. Lett. 32 (2005) L21815.

[10] J. Dumm et al., IceCube coll.: All-Sky Point-Source Search with 40 Strings of IceCube, ICRC0 $9^{2}$ paper 653.

[11] P. Berghaus, IceCube coll.: Muons in IceCube, Proc. XV Int. Symp. on Very High Energy Cosmic Ray Interactions, Paris, France, Sept. 2008, arXiv:0902.0021

[12] D. J. Boersma, L. Gladstone, and A. Karle, Icecube coll.: Moon Shadow Observation by Ice Cube, ICRC09², paper 1173.

[13] R. Abbasi, P. Desiati, and J. C. Vélez, Icecube coll.: Large Scale Cosmic Rays Anistropy With IceCube, ICRC09², paper 1340.

[14] Tibet AS $\gamma$ collaboration: M. Amenomori et al., Science 314 (2006) 439.

[15] Milagro Collaboration: A. A. Abdo et al, Astrophys. J. 698 (2009) 2121.

[16] E. Middell, J. McCartin, and M. D'Agostino, IceCube coll.: Improved Reconstruction of Cascade-Like Events in IceCube, ICRC09 ${ }^{2}$, paper 708.

[17] D. Chirkin, IceCube coll.: Measurement of the atmospheric neutrino energy spectrum with IceCube, ICRC09² ${ }^{2}$, paper 1418.

[18] A. Karle, IceCube coll.: IceCube, ICRC09 ${ }^{2}$, to be published.

[19] L. D. Landau and I. J. Pomeranchuk, Dokl. Akad. Nauk. SSSR 92 (1953) 735 ;

A. B. Migdal, Phys. Rev. 103 (1956) 1811

[20] S. Klein, Rev. Mod. Phys., 71 (1999) 1501

[21] J. Bolmont, B. Voigt, and R. Nahnhauer, IceCube coll.: Very high energy electromagnetic cascades in the LPM regime with IceCube., Proc. 30th Int. Cosmic Ray Conf., Mérida, Mexico, August 2007, arXiv:0711.0353

[22] D. F. Cowen, J. Phys. Conf. Ser. 60 (2007) 227.

[23] K. Mase, A. Ishihara, and S. Yoshida, IceCube coll.: The extremely high energy neutrino search with Ice Cube, ICRC $09^{2}$, paper 861.

2 Proceedings of the 31st International Cosmic Ray Conference, Łódź, Poland, July 2009.
[24] IceCube collaboration: A. Achterberg et al., Phys. Rev. D79 (2009) 102005 .

[25] IceCube collaboration: A. Achterberg et al., Phys. Rev. D76 (2007) 042008 .

[26] IceCube collaboration: A. Achterberg et al., Astrophys. J. 664 (2007) 397, astro-ph/0702265 2 .

[27] A. Silvestri, IceCube coll.: Search for Ultra High Energy Neutrinos with AMANDA, ICRC09² $9^{2}$ paper 549.

[28] S. Razzaque, P. Mészáros, and E. Waxman, Phys. Rev. D68 (2003) 083001.

[29] F. W. Stecker, Phys. Rev. D72 (2005) 107301; A. Mücke et al., Astropart. Phys. 18 (2003) 593.

[30] R. Engel, D. Seckel, and T. Stanev, Phys. Rev. D64 (2001) 093010.

[31] J. Braun et al., Astropart. Phys. 29 (2008) 299.

[32] R. Abbasi et al.,Phys. Rev. D79 (2009) 062001, arXiv:0809.1646

[33] IceCube collaboration: R. Abbasi et al., Astrophys. J. Lett. 701 (2009) L47, arXiv:0905.2253

[34] IceCube collaboration: R. Abbasi et al., Phys. Rev. Lett. 103 (2009) 221102, arXiv:0911.2338

[35] J. L. Bazo Alba, E. Bernardini, and R. Lauer, IceCube coll.: Search for neutrino flares from point sources with IceCube, ICRC09² , paper 960.

[36] M. Baker et al., IceCube coll.: IceCube Time Dependent Point Source Analysis Using Multiwavelength Information, ICRC09² , paper 812.

[37] R. Franke and Elisa Bernardini, IceCube coll.: Neutrino triggered highenergy gamma-ray follow-up with IceCube, ICRC09² , paper 987.

[38] A. Franckowiak et al., IceCube coll.: Optical follow-up of high-energy neutrinos detected by IceCube, ICRC09 ${ }^{2}$, paper 764.

[39] IceCube collaboration: R. Abbasi et al., Astrophys. J. 710 (2010) 346; A. Kappes, P. Roth, E. Strahler, IceCube coll.: Searches for neutrinos from GRBs with the IceCube 22-string detector and sensitivity estimates for the full detector, ICRC09² $9^{2}$ paper 1221.

[40] K. Meagher et al., IceCube coll.: Search for neutrinos from GRBs with Ice Cube, ICRC09 ${ }^{2}$, paper 515.

[41] M. Duvoort and N. van Eindhoven, IceCube coll.: Search for GRB neutrinos via a (stacked) time profile analysis, ICRC09², paper 393.

[42] E. Waxman and J.N. Bahcall, Phys. Rev. Lett. 78 (1997) 2292, arXiv:astro-ph/0211358

[43] IceCube collaboration: R. Abbasi et al., Phys. Rev. Lett. 102 (2009) 201302, arXiv:0902.2460

[44] G. Wikström and J. Edsjö, J. Cosmol. Astropart. Phys. 04 (2009) 009.

[45] P. Gondolo et al., J. Cosmol. Astropart. Phys. 07 (2004) 8.

[46] J. Braun and D. Hubert, IceCube coll.: Searches for WIMP Dark Matter from the Sun with AMANDA, ICRC09² , paper 834, arXiv:0906.1615

[47] D. Hubert, Search with the AMANDA detector for neutralino dark matter in the Sun, Doctoral Thesis, Vrije Universiteit Brussel, May 2009.

[48] IceCube collaboration: R. Abbasi et al., Limits on a muon flux from Kaluza-Klein dark matter annihilations in the Sun from the IceCube 22string detector, arXiv:0910.4480

[49] J. Dunkley et al., Astrophys. J. Suppl. 180 (2009) 306.

[50] J. A. Vandenbroucke et al., Int. J. Mod. Phys. A $21 S 1$ (2006) 259; D. Besson at al., Nucl. Instr. Meth. A 604 (2009) S179.

[51] Freija Descamps, IceCube coll.: Acoustic detection of high energy neutrinos in ice: Status and results from the South Pole Acoustic Test Setup, ICRC09² $^{2}$, paper 1293, arXiv:0908.3251

[52] B. P. Price, J. Geophys. Res., 111 (2006) B02201,

[53] H. Landsman, L. Ruckman, G. S. Varner, IceCube coll., Nucl. Instr. Meth. A 604 (2009) S70.

[54] J. Auffenberg et al., Study of electromagnetic backgrounds in the 25-300 $\mathrm{MHz}$ frequency band at the South Pole, ICRC09², paper 418.

[55] IceCube collaboration, The Design and Physics Capabilities of IceCubes DeepCore Subarray, in preparation.

[56] O. Schultz, S. Euler and D. Grant, IceCube coll.: Implementation of an active veto against atmospheric muons in IceCube DeepCore, ICRC09 ${ }^{2}$, paper 1237.

[57] C. Wiebusch, IceCube coll.: Physics Capabilities of the IceCube DeepCore Detector, ICRC09² , paper 1352, arXiv:0907.2263. D. Grant, D. J. Koskinen and C. Rott, IceCube coll.: Fundamental Neutrino Measurements with IceCube DeepCore, ICRC09² , paper 1336.

[58] O. Mena, I. Mocioiu and S. Razzaque, Phys. Rev. D 78 (2008) 093003, arXiv:0803.3044 